\title{
DOMINANT BALANCE ANALYSIS OF THE FRACTIONAL POWER DAMPED HARMONIC OSCILLATOR
}

\author{
${ }^{1}$ RONALD E. MICKENS ${ }^{1}$ AND 'KALE OYEDEJI ${ }^{2, *}$ \\ ${ }^{1}$ Department of Physics, Clark Atlanta University, Atlanta, GA 30314, USA \\ Stone Mountain, GA USA
}

\begin{abstract}
We apply the principle of dominant balance to determine the solution, for which there is finite time dynamics, in the neighborhood of the extinction of the oscillations, for a simple harmonic oscillator with fractional power damping. This work extends the previous calculations of Mickens $[2,5]$.
\end{abstract}

Key Words: Nonlinear oscillations, finite time dynamics, nonlinear damping.

AMS Subject Classifications: 34C29, 34DOS, 70K25,74H40.

* Contact author

E-mail addresses: rmickens@cau.edu, kaleoyedeji@comcast.net 


\section{Introduction}

The simple harmonic oscillator ( $\mathrm{SHO}$ ) with cube root damping

$$
\ddot{x}+x=-\in(\dot{x})^{\frac{1}{3}}, \quad \in>0
$$

has been investigated by Mickens and his collaborators [2,5], with a generalization to Duffing type nonlinear oscillators [4]. The general dynamics of Eq.(1.1) has been discussed in Mickens [2]. In summary,

(i) In the $x-y$ phase-plane, the system of differential equations are

$$
\frac{d x}{d t}=y, \quad \frac{d y}{d t}=-x-\in y^{\frac{1}{3}} .
$$

(ii) There is a single fixed-point

$$
(\bar{x}, \bar{y})=(0,0)
$$

and it is (globally) stable.

(iii) In general, the solutions oscillate with decreasing amplitude to the fixed-point.

(iv) For $0<\epsilon \ll 1$, the application of perturbation theory, i.e., the method of averaging, shows that the system has finite-time dynamics. This means that there exists a time, $t^{*}$

$$
t^{*}=f\left(\in, x_{0}\right\}
$$

with initial conditions

$$
x_{0}=x(0), \quad \dot{x}(0)=y(0)=0,
$$

such that

$$
x(t)=0, \quad t \geq t^{*} .
$$

(v) There are two time scales associated with this system. The natural periodic oscillation of the SHO, i.e., Eq.(1.1) with $\in=0$,and the time scale of the damping. These are, respectively,

$$
T_{S H O}=2 \pi, \quad T_{0}=\left(\frac{x_{0}^{\frac{2}{3}}}{\epsilon}\right)^{\frac{3}{5}},
$$

where

$$
T_{S H O} \ll T_{0}, \quad 0<\in \ll 1 .
$$

This short note has two goals: First, generalize the damping to any fractional power of the damping term, i.e., Eq.(1.1) becomes

$$
\ddot{x}+x=-\in(\dot{x})^{p}, \quad \in>0,
$$

and

$$
0<p<1
$$


Second, calculate the analytic form of the solution to Eq.(1.9) in the neighborhood of its extinction time $t_{p}^{*}$. (Note that the mathematical form of the dominant balance anzat incorporates the assumptions of a finite time extinction.)

In outline, Section 2 provides preliminary information and references to time scales and the dominant balance method, which is then used in Section 3 to evaluate the required(asymptotic) solution. We summarize our conclusions in Section 4 .

\section{Preliminaries:}

To eliminate the need for absolute value evaluations and other related issues, we select $\mathrm{p}$ to be

$$
p=\frac{2 m+1}{2 n+1} ; \quad(m . n): 0,1,2,3, \ldots, \text { with } 0 \leq m<n,
$$

and this gives the restriction of Eq. (1.10). We call these fractions "odd" , in the sense that $x^{p}$ is an odd function, i.e., it can be shown that

$$
(-x)^{p}=-x^{p} .
$$

Note that $q=1-p$ is an "even" fraction.

The method of dominant balance is a technique for formulating and calculating the asymptotic behavior of differential and many other many other types of equations. See Mickens [3] for a summary of this methodology along with references to its applications.

\section{Dominant Balance Analysis:}

The use of the averaging method on

$$
\ddot{x}+x=-\in(\dot{x})^{p}, \quad 0<p<1,
$$

shows that the solutions have properties similar to the particular case $p=\frac{1}{3}$, i.e., the solutions become zero in a finite time. Thus, we take the dominant balance ansatz to be

$$
x(t)=\left\{\begin{array}{c}
A\left(t^{*}-t\right)^{\beta}, \quad t^{*}-\delta<t \leq t^{*} \\
0, \quad t>t^{*}
\end{array}\right.
$$

for some $\delta>0$ and for constants $(A, \beta)$ to be determined. Note that $t^{*}$ can not be determined by this procedure since the differential equation is invariant under $t \rightarrow t+t_{0}$, where $t_{0}$ is arbitrary.

Substituting Eq.(3.2) into Eq. (3.1) gives

$$
\beta(\beta-1) A\left(t^{*}-t\right)^{(\beta-2)}+A\left(t^{*}-t\right)^{\beta} \backsim \in \beta^{p}\left(t^{*}-t\right)^{(\beta-1) p} .
$$

For the balancing there are three possibilities:

(I)

$$
\beta(\beta-1) A\left(t^{*}-1\right)^{(\beta-2)}+A\left(t^{*}-t\right)^{\beta} \sim 0,
$$


(II)

$$
\begin{gathered}
A\left(t^{*}-t\right)^{\beta} \backsim \in \beta^{p} A^{p}\left(t^{*}-t\right)^{(\beta-1) p}, \\
\beta(\beta-1) A\left(t^{*}-t\right)^{(\beta-2\}} \backsim \in \beta^{p} A^{p}\left(t^{*}-t\right)^{(\beta-1\} p} .
\end{gathered}
$$

Equating the coefficients and powers gives

$$
\beta-2=\beta \text { or no solution possible; }
$$

$$
\beta=(\beta-1) p \quad \text { or } \quad \beta=-\left(\frac{p}{1-p}\right)<0
$$

For this case, there is a singularity at $t=t^{*}$, consequently, the solution is unbounded. Thus, this case can be ruled out.

(III) $\beta-2=(\beta-1) p$ and $\beta(\beta-1) A=\in \beta^{p} A^{p}$ and, we calculate $\beta$ and $A$ to be

$$
\beta=\frac{2-p}{1-p}>1, \quad A=\left(\frac{1}{\beta}\right)\left(\frac{\epsilon}{\beta-1}\right)\left(\frac{1}{1-p}\right) .
$$

Next, we need to check that the following two conditions hold

$$
\beta>\beta-2, \quad \beta>(\beta-1) p,
$$

i.e., the neglected term must smaller as $t \rightarrow t^{*}$, from below $t^{*}$. A simple calculation shows both inequalities hold.

Putting these results together gives the following representation of the solution, $x(t)$, in a neighborhood of $t^{*}$ :

$$
x(t) \backsim\left\{\begin{array}{c}
A\left(t^{*}-t\right)^{\beta}, \quad t^{*}-\delta<t \leq t^{*}, \delta>0, \\
0, \quad t>t^{*},
\end{array}\right.
$$

where $(A, \beta)$ are given in the expressions of Eq. (3.8).

\section{Discussion}

Combining the results of Mickens[2,5], we conclude that the SHO with nonlinear, frictional power damping has finite-time dynamics. Further, we have determined a mathematical expression for the solution near the extinction time $t^{*}$; see Eq.(3.10). Thus, the motion of this oscillator is decreasing and oscillatory $[2,5]$.

Finally, it should be indicated that our results are not in agreement with those obtained by Amann and Diaz [1]. We have examined their paper [1], but can not follow their arguments. 


\section{Acknowledgement}

REM thanks Professor Ivana Kovacic, University of Novi Sad, for maintaing his interest in nonlinear oscillations and, in addition, for her outstanding skills for resolving many issues related to nonlinear dynamics.

\section{References}

[1] H. Amann, J.I. Diaz, A note on the dynamics of an oscillator in the presence of strong friction, Nonlinear Analysis: Theory, Methods and Applications 55 (2003) 209-216.

[2] R.E. Mickens, Calculation of order $\in$ dynamics for an NSFD discretization of a cube root damped oscillator, Journal of Difference Equations and Applications 20 (2014) 826-836.

[3] R. E. Mickens, Mathematical Methods for the Natural and Engineering Sciences. $2^{\text {nd }}$ edition, World Scientific, Singapore, 2017. See section13.10.

[4] R. E. Mickens, R. Bullock, W.E. Collins, K. Oyedeji, Nonlinear-damped Duffing Oscillator having finite time dynamics, arXiv: 1404.5596v1 [Nlin.CD] 22 April 2014.

[5] R. E. Mickens, K. Oyedeji, S.A. Rocker, Analysis of the simple harmonic oscillator with fractional damping, Journal of Sound and Vibration, 268 (2003) 839-842. 